\title{
Comparative of physical complaints, depression and OCD in people with hearing impaired and normal people in Ahwaz "Iran"
}

\author{
Mojtaba Tavakoli ${ }^{1}$, Elham Aghaie ${ }^{2}$, Sahar Shomeil Shushtari ${ }^{1}$, Azam Aghaei ${ }^{1}$, Amene Kuhi ${ }^{1}$, \\ Behruz Mahmoodi Bakhtiari ${ }^{3}$ \\ ${ }^{1}$ Department of Audiology, Musculoskeletal Research Center, Jundishapur University of Medical Sciences, Ahwaz, Iran \\ ${ }^{2}$ Faculty of Humanistic Sciences, Shahed University, Tehran, Iran \\ ${ }^{3}$ Department of Performing Arts, University of Tehran, Iran
}

\section{Email address:}

mojtaba.tavakoli@gmail.com (M. Tavakoli),elhamaghaie87@yahoo.com (E.Aghaie), sshomeil@gmail.com(S. S. Shushtari), azam.aghaie@yahoo.com (A. Aghaei), kuhi_amene@yahoo.com (A. Kuhi), Mbakhtiari@ut.ac.ir (B. M. Bakhtiari)

\section{To cite this article:}

Mojtaba Tavakoli, Elham Aghaie, Sahar Shomeil Shushtari, Azam Aghaei, Amene Kuhi, Behruz Mahmoodi Bakhtiari. Comparative of Physical Complaints, Depression and OCD in People with Hearing Impaired and Normal People in Ahwaz "Iran". Psychology and Behavioral Sciences. Vol. 3, No. 4, 2014, pp. 126-130. doi: 10.11648/j.pbs.20140304.14

\begin{abstract}
Hearing is an important sense. Some parts of our communication with the outside world are based on this sense. Those who are deaf from birth cannot acquire language. Social reactions and the type of behaviors with that people who are faced and also showing sympathy to them or blaming them is some causes of mood disorders among these people. This study sought to compare the psychological disorders of people having normal hearing and those suffering from hearing loss in Ahwaz. Method: This study has a comparative nature. Participants of this study were 40 normal-hearing and 40 people who suffered from hearing loss. The data were collected on the basis of SCL-90-R1 questionnaire, which is one of the most-applied questionnaires in psychiatry, and a demographic questionnaire. Results: The findings of this study showed an observable difference between people having normal hearing and those who suffer from hearing loss in terms of psychological disorders for instance (physical problems, depression, obsessive-compulsive disorder). The results showed that such disorders are more common among people who suffer from hearing loss. Conclusion: There are meaningful differences between people who have normal hearing and those suffering from hearing impaired in terms of all main variables of the study.
\end{abstract}

Keywords: Physical Complaint, Depression, Obsessive-Compulsive Disorder, Hearing Impaired

\section{Introduction}

Hearing-impairment reduces life quality. It might lead to isolation, social inactivity, and a sense of rejection. Audition has an undeniable role in character's improvement and socio-psychological developments [1]. Social reactions and showing behaviors such as sympathy or blame are the causes of some mood disorders among this group of people. Although society pays more attention to sight problems than auditory problems, the problems of hearing-impaired people is much greater than the problems of sight-impaired ones. It has been said that suicide is more prevalent among those who suffer from hearing loss than those who suffer from sight problems. The consequences related to auditory disorders show themselves in different problem areas such as individual problems (delay in speaking, isolation, pessimism, irritation), school problems (excessive inactivity and inability to cope with courses at school), psychological problems (depression, anxiety, hallucination), and social problems (unsocial and anti-social reactions, revengefulness) [2]. Those people who suffer from hearing loss are more prone to psychological disorders, because they are unable to connect with others and they might be dominated by a feeling of inefficiency. Hearing-impairment can lead to isolation, social inactivity, a feeling of rejection, and a low-quality life [1]. A number of studies which were conducted in 1956 in US showed that hearing loss is the most common physical disorder among people and $\% 7$ of people living in this country suffered from such problems. 
According to a report issued by US demographic association, one out of a thousand children suffer from sever hearing impaired. Less severe hearing problems are observed between 6-16 people out of 1000 cases. Various studies have shown that hearing-impaired people suffer from psychological problems and they are paid insufficient attention by the society. So, this study seeks to compare the psychological conditions among these people and those who have normal hearing.

In a study conducted by Mirzaieasl (2000), the psychological conditions of people suffering from hearing loss and people having normal hearing were compared. They were selected by multi-stage cluster-sampling method. These participants were examined in terms of depression and social and physical problems. The results indicated that there is a meaningful difference between the psychological conditions of people suffering from hearing loss and those who have normal hearing. Also, the results suggested that there were no meaningful difference between people in terms of social relation problems and physical disorders [3].

Richard and Chu (2009) found that mothers of hearing-impaired children suffer from sleep disorder, depression, anxiety, and stress [4]. Aliakbari et al (2011) reported that mothers of mentally-retarded children suffer from anxiety problems compared to mothers of normal children [5]. In a study conducted by Kohansal (2008) in Shiraz, social skills of deaf and hearing school girls (15-18 years old) were compared. In this study, 106 school girls (53 hearing and 53 deaf students) were selected randomly. Matson questionnaire of social skills was given to these students. The results showed that hearing students were in a better position in terms of social skills, self-confidence, and anti-social behaviors. But, in terms of aggressive behaviors and relation with peers, no meaningful difference was observed between the two groups [6].

In a study conducted by Hussein Abadi et al (2007), the relationship between hearing aids and depression was investigated among elderly people in one centre in Tehran. In this study, the level of depression among three groups of elderly people (30 elderly people having normal hearing, 30 elderly people suffering from moderately severe hearing loss without hearing aid, 20 elderly people suffering from moderately severe hearing problems who were using hearing aid). Beck Depression Inventory (BDI-II) was used to evaluate the level of depression among these three groups of participants. The data were analyzed by a one-way analysis of variance. The mean of depression scores were 1143, 2153, and 1640 for these groups respectively. This shows a meaningful difference among these three groups. No correlation was found between age and depression among the participants who were suffering from hearing loss without using hearing aids. But, among the other two groups, the correlation was observable [7].

In a study conducted by Gholam Reza Afrooz (2009), the socio-psychological and cognitive characteristics were analysed and compared among young blinds and young deaf people. Because light, sound, vision, and audition have various influences on the development of character, some special characteristics of these people have been discussed. He notes that lack of hearing not only has a major impact on learning ability and cognitive developments, but also is a cause of depression among these people [1].

In a study by Raffael Boy (2012), the relationship between hearing loss and depression was investigated among elderly people. In this study, 15 elderly people who were over 70 years old were examined. These participants were suffering from hearing loss and depression. General health, level of satisfaction with life, social skills, emotional stability, and psychological health of these participants were investigated by a psychological questionnaire. These tests were conducted with 1,3 , and 6 month gaps by programmable hearing aids (digital double piece). At the beginning of using hearing aids, depression was reduced and life quality improves [8].

In a study made by Flinger (2007), hard of hearing participants were examined in terms of anxiety and life quality. In this study, WHO life quality questionnaire was given to 373 hard of hearing participants. Hard of hearing participants, compared to hearing ones, received lower scores in their social relations. They might be more isolated in their relations [9].

In a research conducted by Hinsee et al (2002), relational and psychological consequences of sensorineural hearing loss was studied among elderly people. It was revealed that coping with sensorineural hearing loss is very difficult for elderly people and it can lead to problems such as depression, anxiety, inactivity, and social dissatisfaction. Sensorineural hearing loss has a negative impact on social and psychological operations and it might reduce life quality [10].

In a study made by Fariba Yazd Khasti and Ahmad Yarmohammadian (2009) in Isfahan, the relationship between depression among mothers and emotional intelligence of their daughters (hearing and deaf) was investigated. The results showed that loss of hearing among daughters was correlated with depression among mothers. Also, there was a correlation between depression among mothers and emotional intelligence of daughters. Because of negative emotional relations, a high level of depression had a direct correlation with low emotional intelligence. A low level of depression among mothers had a direct correlation with emotional intelligence among deaf teenagers [11].

In a study conducted by Pardis Alivand et al (2012), the psychological health of elderly people who were suffering from hearing loss was examined in Ahwaz. Seventy two 60 -year-old or older people were examined in Imam Khomeini hospital of Ahwaz. Thirty participants were using hearing aid and 30 participants were not using them. The Goldberg General Health Questionnaire (GHQ), which includes 91 items, was used (including questions related to psychological health and four sub-indices related to physical problems, anxiety, disorder in social skills, and depression. The findings of this study showed that those elderly people who used hearing aids had a better psychological condition compared to those who were suffering from hearing loss and did not use hearing aids [12]. 


\section{Objectives}

1. A comparison between people suffering from hearing loss and those who have normal hearing in terms of psychological disorders.

2. A comparison between people suffering from hearing loss and those who have normal hearing in terms of physical complaint.

3. A comparison between people suffering from hearing loss and those who have normal hearing in terms of depression levels.

4. A comparison between people suffering from hearing loss and those who have normal hearing in terms of obsessive-compulsive disorder (OCD).

\section{Method}

This research was a comparative study. Participants of the study were two groups. The first group consisted of 40 people who had no hearing problems. The second group consisted of 40 people who were suffering from hearing loss. These participants were using hearing aids. Hearing aids had been provided by the association of deaf and auditory measurement clinics in Ahwaz. Sampling was multi-stage and random. Two questionnaires were used to collect the data: (1) SCL-90-R Inventory (2) Demographic questionnaire [13].

Those participants who were suffering from hearing loss went to the association of deaf or auditory measurement clinics to answer questionnaire items. They were provided with necessary guidance by the trainers to answer the items. The collected data were analysed by SPSS16 software.

\section{Results}

There is a meaningful difference between those who suffer from hearing loss and those who have a normal hearing in terms of depression scores (14.02 compared to 7.52). This shows that depression is more common among people who suffer from hearing loss.

There is a meaningful difference between those who suffer from hearing loss and those who have a normal hearing in terms of obsessive-compulsive disorder scores (9.72 compared to 5.57). This shows that obsessive-compulsive thoughts are more common among people who suffer from hearing loss.

There is a meaningful difference between those who suffer from hearing loss and those who have a normal hearing in terms of physical complaints scores (11.80 compared to 5.30). This shows that physical complaints are more common among people who suffer from hearing loss.

Table 1. Central tendency and dispersion indices of psychological disorder score among people who suffer from hearing loss those who have normal hearing

\begin{tabular}{lllllll}
\hline & \multicolumn{3}{l}{ People suffering from hearing loss } & \multicolumn{3}{l}{ People who have normal hearing } \\
\cline { 2 - 7 } & NO. & Mean & Standard deviation & NO. & Mean & Standard deviation \\
\hline Depression & 40 & 14.02 & 10.3 & 40 & 7.52 & 6.9 \\
Obsessive-compulsive thoughts & 40 & 9.72 & 5.4 & 40 & 5.57 & 5.3 \\
Physical complaints & 40 & 11.80 & 8.4 & 40 & 5.30 & 4.7 \\
\hline
\end{tabular}

\section{Examination of First Objective}

Table 2. The results of multivariate analysis of co-variance (MANCOVA) for psychological disorder scores among people who suffer from hearing loss and those who have normal hearing

\begin{tabular}{|c|c|c|c|c|c|c|c|}
\hline test & value & F test & $\begin{array}{l}\text { Degree of freedom of } \\
\text { hypothesis }\end{array}$ & $\begin{array}{l}\text { Degree of freedom of } \\
\text { error }\end{array}$ & Level of meaningfulness & Eta $^{2}$ & Statistical power \\
\hline Pillai's trace & 0.215 & 2.106 & 9.000 & 69.000 & 0.041 & 0.215 & 0.834 \\
\hline$\lambda$ wilks & 0.785 & 2.106 & 9.000 & 69.000 & 0.041 & 0.215 & 0.834 \\
\hline Hitling trace & 0.275 & 2.106 & 9.000 & 69.000 & 0.041 & 0.215 & 0.834 \\
\hline $\begin{array}{l}\text { Roy's largest } \\
\text { root }\end{array}$ & 0.275 & 2.106 & 9.000 & 69.000 & 0.041 & 0.215 & 0.834 \\
\hline
\end{tabular}

Table 3. The results of the one-way multivariate analysis of co-variance (MANCOVA) for psychological disorder scores (physical complaints, depression, obsessive -compulsive disorder) among people suffering from hearing loss and those having normal behavior.

\begin{tabular}{|c|c|c|c|c|c|c|c|c|}
\hline & & $\begin{array}{l}\text { Sum of } \\
\text { squares }\end{array}$ & $\begin{array}{l}\text { Degree of } \\
\text { freedom }\end{array}$ & $\begin{array}{l}\text { Mean of } \\
\text { squares }\end{array}$ & F test & $\begin{array}{l}\text { Level of } \\
\text { meaningfulness }\end{array}$ & $\mathrm{Eta}^{2}$ & $\begin{array}{l}\text { Statistical } \\
\text { power }\end{array}$ \\
\hline \multirow{3}{*}{$\begin{array}{l}\text { Psychological } \\
\text { disorder }\end{array}$} & depression & 619.572 & 1 & 619.572 & 7.836 & 0.006 & 0.092 & 0.789 \\
\hline & $\begin{array}{l}\text { Obsessive-compulsive } \\
\text { disorder }\end{array}$ & 269.450 & 1 & 269.450 & 9.148 & 0.003 & 0.106 & 0.848 \\
\hline & Physical complaints & 608.338 & 1 & 608.338 & 12.90 & 0.001 & 0.144 & \\
\hline
\end{tabular}


As can be seen in table 2, level of meaningfulness is 0.05 for all tests. So, there is at least one meaningful difference between people suffering from hearing loss and those who have normal hearing in terms of psychological disorder scores (physical complaints, depression, obsessive-compulsive disorder). The value of $\lambda$ Wilks test is equal to $0.785(\mathrm{~F}=2.106)$. It shows a meaningful difference between people suffering from hearing loss and those who have normal hearing in terms of psychological disorder scores (physical complaints, depression, obsessive-compulsive disorder $)(p=0.05)$. Also, Eta2 is equal to 21 percent. In other words, 21 percent of individual differences in terms of psychological disorders are related to differences between two groups. The one-way multivariate analysis of co-variance was used to investigate differences between people suffering from hearing loss and those having normal hearing in terms of psychological disorder scores (physical complaints, depression, obsessive-compulsive disorder). The results have been presented in table 2 .

As can be seen in table 3 , the F test shows that there is a meaningful difference between people suffering from hearing disorder and those having normal hearing $(p=0.05)$ in terms of psychological disorder scores (physical complaints, depression, obsessive-compulsive disorder). So, null hypothesis is rejected.

\section{Discussion and Conclusion}

Psychological reactions of hard of hearing people might be similar to the reactions of those people suffering from psychological tensions. Because these people cannot either hear or properly hear, they are unable to recognize words and sentences. So, they are placed in an uncertain condition. This can be the cause of anxiety and obsession which might lead to other psychological problems. The data of the present study showed that with a certainty of $\% 95$, there is meaningful difference between who suffer from hearing loss and those having a normal hearing in terms of psychological disorder scores (physical complaints, depression, obsessive-compulsive disorder). The mean of such problems is lower among people having normal hearing. As can be seen in table 3, compared to people who have a normal hearing, those who suffer from hearing loss are more prone to psychological disorders (physical complaints, depression, obsessive-compulsive disorder).

\subsection{A Comparison between People Suffering from Hearing Loss and those who have Normal Hearing in Terms of Physical Complaints}

In this study, it was hypothesized that physical complaints are more common among people suffering from hearing loss. The data of this study confirmed this hypothesis. A meaningful difference was observed between people having normal hearing and those suffering from hearing loss $(\mathrm{p}<0.001)$.

\subsection{A Comparison between People Suffering from Hearing Loss and those who have Normal Hearing in Terms of Obsessive-Compulsive Disorder}

In this study, it was hypothesized that obsessive-compulsive disorder is more common among people suffering from hearing loss. The data of this study confirmed this hypothesis. A meaningful difference was observed between people having normal hearing and those suffering from hearing loss $(\mathrm{p}<0.003)$.

\subsection{A comparison between People Suffering from Hearing Loss and those who have Normal Hearing in Terms of Depression Level}

In this study, it was hypothesized that depression is more common among people suffering from hearing loss. The data of this study confirmed this hypothesis. A meaningful difference was observed between people having normal hearing and those suffering from hearing loss $(\mathrm{p}<0.006)$.

\section{References}

[1] A. Gh. Comparison of analytical and psychological principles of cognitive and psycho-social characteristics of blind and deaf people. Journal of Psychology Thirty-Nine Years, No 2, 1388, pp. 1 -

[2] Milanifar B. Psychology of Exceptional Children, Sixteenth Edition, 2005

[3] Mirzaie Asl, M. Comparison of mental health between groups of hearing impaired people and those with normal hearing. October 2000.

[4] Chu, Richdale AL. sleep quality and psychological wellbeing in mothers of children with developmental disabilities. Res Dev Disablil. 2009 : 30 (6) : 1512-22.

[5] Aliakbari Dehkordi M, Kakojoibari AA, Mohtashami T, Yektakhah S. stress in mothers of hearing impaired children compared to mothers of normal and other disabled children. Audiol. $2011: 20$ (1): 128- 36.

[6] Kohansal.H. Comparison of social skills in impaired students female and normal students 18-15 year old in Shiraz, 2008

[7] Hossein-Abadi R., Gourabi K., Share H. Impact of hearing aids on depression in the elderly with in elderly centers in Tehran. Elder, 2007, 2 (3): 9-205.

[8] Raffaella Boi,1 Luca Racca,2 Antonio Cavallero,3 Veronica Carpaneto,1 Matteo Racca,2 Francesca Dall' Acqua,1 Michele Ricchetti,2 Alida Santelli3 and Patrizio Odetti1. Hearing loss and depressive symptoms in elderly patients. Geriatr Gerontol Int 2012; 12: 440-445

[9] Fellinger J, Holzinger D, Gerich J, Goldberg D. Mental distress and quality of life in the hard of hearing. Acta Psychiatr Scand. 2007 Mar;115(3):243-5.

[10] Heine C, Browning CJ. Communication and psychosocial consequences of sensory loss in older adults: overview and rehabilitation directions. Disabil Rehabil. 2002 Oct 15;24(15):763-73. 
[11] Yazdkhasti F., Yar Mhdyan.A. compare the relationship between maternal depression and emotional intelligence hearing and deaf teenage girls

Isfahan, Iran, 2001 Journal of Behavioral Sciences

[12] Alivand.P. Zahir M. Ghasem-Zadeh,R Latif, Fathi.S.M.Fathi.K. Comparing the mental health of older adults with hearing aids and without hearing aids of Ahwaz in 2012, the Journal of Allied Health and Rehabilitation Sciences, Mashhad - Volume 3 - Issue 1( Spring \& Summer 2014 ).

[13] Fathi Ashtiani A., Dastani.M. Psychological tests, 2011. 